\title{
ESSAI D'INCUBATION SOUS GRAVIERS EN BASSIN CIRCULAIRE POUR LA PRODUCTION DE GENITEURS DE SAUMONS DE FONTAINE
}

\author{
Par Richard VIBERT \\ Ingénieur des Eaux et Forêts. \\ (Service des Recherches Piscicoles).
}

Les acclimatations de Saumons de fontaine (Salvelinus fontinalis) faites par l'Inspecteur général des Eaux et Forêts LARrieu, de 1925 à 1939, dans les eaux pyrénéennes, ont donné pleine satisfaction. Dans nombre de rivières d'altitude, vierges ou peu peuplées, cette espèce s'est bien développée et a fourni aux pêcheurs des poissons de bon sport. Très voraces, ces poissons finissent par être presque tous pris par les pêcheurs, ce qui est à la fois un avantage et un inconvénient, ce qui oblige, en tout cas, à maintenir les repeuplements.

Pendant la guerre et les années d'occupation, notre cheptel de reproducteurs tomba à peu près à zéro. Nous avons donc pensé qu'il serait utile de remonter un cheptel de reproducteurs de Saumons de fontaine à la pisciculture domaniale de Lees-Athas, pour l'approvisionnement en œufs et alevins des Sociétés de Pêche pyrénéennes.

Deux essais furent tentés parallèlement sans succès :

- Acquisition d'un lot de Saumons de fontaine de deux ans de la pisciculture d'Étrun (Pas-de-Calais). Presque tous ces géniteurs furent pris de nuit par des braconniers dans les petits étangs à eau renouvelée de la pisciculture qui, faute de crédits, n'étaient pas encore clos ;

- Importation d'œufs de Saumons de fontaine du Danemark, incubation et alevinage dans la pisciculture de Lees-Athas donnèrent des résultats catastrophiques : 100.000 œufs fournissaient à grand peine 20.000 alevins malingres, dont la moitié mouraient pendant les transports aux lieux de déversement.

En Novembre 1950, les quelques géniteurs qui nous restaient nous fournirent environ 5.000 œufs, sur lesquels une énorme mortalité commença à s'abattre, comme les années précédentes. 2.500 à 3.000 œufs arrivèrent au stade d'embryonnement en Décembre 1950.

Ces oufs furent alors mis dans un tas de gravier placé dans un bassin circulaire à alimentation d'eau tangentielle. Ce bassin était du type 
standard américain : 8 mètres de diamètre -30 centimètres d'eau sur les bords - 60 centimètres d'eau au milieu - débit 3 litres-seconde sous $1 \mathrm{~m}$. 50 de pression d'eau. Le bassin était recouvert d'un grillage contre les martins-pêcheurs et les merles d'eau, très abondants en cet endroit.

En Janvier-Février 1951 les alevins, ayant résorbé leur vésicule, sortirent du tas de graviers. Ils paraissaient assez nombreux, quoique bien petits et bien perdus dans ces quelques 50 mètres carrés. Le nourrissage commença, exclusivement au "Vorax ", aliment complet fourni par la Société F.A.P.A. La croissance parut normale. Aux mois d'Août-Septembre, 10 a $15 \mathrm{kgs}$. d'asticots, provenant de rates avariées, furent ajoutés à la nourriture.

Le 20 Novembre 1951, le bassin dut être vidé à l'occasion de travaux sur les conduites d'eau. La pêche donna :

2.180 Saumons de fontaine récupérés vivants;

30 à 40 Saumons de fontaine asphyxiés dans la vase en fin d'opération;

80 à 100 Saumons de fontaine échappés en fin d'opération.

Au total, en chiffre rond : 2.300 Saumons de fontaine.

Les 2.180 Saumons vivants furent mis dans un bassin de stockage. Un échantillonnage de 500 individus de toutes tailles, pris le 12 Décembre 1951, donna un poids moyen de 13 gr. 7 - les extrêmes variant, à vue d'œil, entre 5 et 80 grammes.

Au total, la production dépassa donc nettement $30 \mathrm{kgs}$. de " yearlings", à partir de 2.500 à 3.000 œufs, et cela avec 10 à 15 kgs. d'asticots et 160 à 180 kgs. de "Vorax ", nourriture qui n'est cependant étudiée que pour la nourriture des jeunes alevins.

$\mathrm{Au}$ début de 1952, ces "yearlings " furent remis en bassin circulaire, habitués à se nourrir de poisson de mer frais, puis déversés dans un petit étang à eau renouvelée. Là, ils furent nourris chaque jour au poisson de mer et pouvaient trouver en outre, sur place, un appoint non négligeable de nourriture naturelle. En Octobre 1952, à la suite d'une forte crue du ruisseau d'alimentation et de fuites aux vannes, l'étang déborda pendant plusieurs jours. Il fut impossible de savoir si les fuites de poissons provoquées par la crue furent importantes. Fin Novembre 1952, l'étang fut mis à sec et donna 647 Saumons de fontaine (475 femelles et 172 mâles) pesant $57 \mathrm{kgs.} 1 / 2$, soit un poids moyen de 89 grammes. Comme il fallait s'y attendre, une partie des femelles, âgées de deux ans seulement, arrivaient déjà à maturité sexuelle. Cette première ponte, précoce, fournit 60.000 œufs.

On peut donc espérer que, sauf imprévu, la pisciculture de Lees-Athas, à eaux froides, pourra dès l'année prochaine commencer à fonctionner comme centre de production d'œufs de Saumons de fontaine.

Nous pensons rendre service en signalant dès à présent les conclusions qui, dans les conditions de l'expérience, semblent devoir être retenues :

$1^{0}$ La mortalité habituelle et intense sur les cufs de Saumons de fontaine à la pisciculture de Lees-Athas, a été arrêtée dès que les œufs ont 
été mis sous graviers, dehors, dans des conditions se rapprochant des conditions naturelles;

$2^{\circ}$ Le taux de survie au bout de onze mois : 2.300 yearlings sur 2.500 à 3.000 œufs, mais sans aucun triage, montre que le cannibalisme a été nul ou insignifiant. D'autant plus que, à l'exclusion des oiseaux empéchés par le grillage, quelques décès sont certainement dus à trois ou quatre Truites arrivées par les conduites d'eau;

$3^{\circ}$ En onze mois, et malgré une surveillance attentive, aucun mort n'a été aperçu, ce qui dénote un excellent état sanitaire que l'on pourrait presque qualifier d'anormal;

$4^{0}$ Poursuivi pendant dix mois consécutifs, et de façon exclusive, à l'exception de l'adjonction d'asticots en Aoùt-Septembre, le nourrissage à l'aliment complet "Vorax " n'a donné aucun déboire, bien au contraire ;

$5^{\circ}$ Avec les eaux froides de Lees-Athas (source $8^{\circ}$ été comme hiver température ne dépassant pas $11^{\circ}$ au centre du bassin circulaire en Août) ce bassin a pu rester onze mois en eau sans le moindre nettoyage. Le seul inconvénient a été que vase et déchets organiques accumulés au centre ont fait perdre quelques poissons à la pêche. Cette masse de vase et de déchets organiques a-t-elle été le centre de formation de vitamines diverses comme cela se passe dans le fumier ? Ces vitamines ont-elles passé dans les algues qui se sont développées avec abondance dans ce bassin ? Ces algues ont-elles été ingérées directement ou indirectement par les poissons, leur apportant un appoint de vitamines inexistant dans les bassins trop propres, et contribué ainsi à leur rusticité ? Il est trop tôt encore pour l'affirmer. 